\title{
Un estado del arte sobre la inclusión y el acceso de la migración a la salud pública argentina
}

\section{A state of the art on the inclusion and access of migration to Argentine public health}

\section{Angerlin Rangel Mujica}

Facultad Latinoamericana de Ciencias Sociales FLACSO, Argentina

rangelangerlin@gmail.com

\section{Resumen}

Este artículo analiza algunas de las dimensiones del concepto de inclusión, con el fin de definir el contexto en el cual se desarrolla el acceso de los inmigrantes a la salud pública en Argentina, país protagonista por las nuevas dinámicas migratorias que emergieron en el escenario suramericano a partir del 2015. Mediante un análisis documental sobre el abordaje del sistema de protección e inclusión social del inmigrante, se presenta una revisión de fuentes con énfasis en el binomio entre migración y salud. Estas categorías funcionan como indicadores que describen un vínculo tenso y en constante mutación; es el vínculo entre lo nacional y lo extranjero, entre lo regular o lo irregular, y entre inclusión y acceso efectivo a derecho.

Palabras clave: inclusión, protección social, migración, salud pública.

\begin{abstract}
This article analyzes some of the dimensions of the concept of inclusion, in order to define the context in which the access of immigrants to public health develops in Argentina, a leading country due to the new migratory dynamics that emerged in the South American scenario. from 2015. Through a documentary analysis on the approach to the system of protection and social inclusion of the migrant, a review of sources is presented with emphasis on the binomial between migration and health. These categories function as indicators that describe a tense and constantly changing bond; it is the link between the national and the foreign, between the regular or the irregular, and between inclusion and effective access to law.
\end{abstract}

Keywords: inclusion, social protection, migration, public health.

Articulo: Recibido el 10 de septiembre de 2021 y aprobado el 13 de octubre de 2021

Cómo citar este artículo:

Rangel Mujica, A. (2021). Un estado del arte sobre la inclusión y el acceso de la migración a la salud pública argentina. Reflexión política 23(48), pp. 40-54 doi: https://doi.org/10.29375/01240781.4238

\section{Introducción}

Se presentan los resultados preliminares de una investigación que planteó analizar la inclusión de la inmigración venezolana a la salud pública en Argentina desde el 2015 hasta hoy: un estudio de caso sobre la Ciudad Autónoma de Buenos Aires (CABA). El estudio se introduce en el debate sobre migración e inclusión a la salud pública; inclusión entendida como "el acceso de los migrantes a los derechos sociales, en igualdad de condiciones y sin discriminación” (OIM, 2017; Ferrajoli, 2006). Sobre este marco, 
el objetivo del artículo es presentar, de manera sintética, un relevamiento de fuentes que responden a cómo es el tratamiento académico y político que ha tenido el tema "migración, salud pública, garantía y goce de derechos" en Argentina; el análisis se hace en el marco de los cambios que imponen las dinámicas globales en las agendas políticas nacionales (Domenech y Pereira, 2017; Gíl y Santi, 2019).

Se realizó una recopilación de investigaciones empíricas publicadas en plataformas como Academia.edu, Redalyc, RefSeek y Scielo entre el 2000 y el 2021. Se utilizaron y combinaron las palabras clave inclusión, protección social, migración y acceso a la salud de la población migrante en Argentina. Asimismo, se revisó la base legal Infoleg para sumar el aspecto normativo a algunas reflexiones, tomadas de documentos publicados por organismos internacionales sobre la gobernanza de la migración en sus ámbitos global, regional, nacional y local. Finalmente, se triangularon los resultados de los referentes documentales, entre artículos de investigación, disertaciones teóricas y normas consultadas para presentar el análisis en cuatro ejes: 1.-génesis del concepto de inclusión; 2.-síntesis del marco jurídico sobre la protección social al inmigrante; 3.-inclusión de la migración a la salud pública en Argentina y 4.-una breve caracterización del sistema de salud pública argentino.

\section{Génisis y transformación del concepto de inclusión}

Para iniciar la reflexión sobre la inclusión, es pertinente tomar la exploración conceptual que realiza Camarero (1998), en la cual hace un recuento cronológico del origen de su definición. Este autor señala que es en Europa en donde se dan los principales indicios por conceptualizarla y por tratar de explicarla a partir de la noción de exclusión, definición que se remonta a la Francia de los sesenta y setenta cuyos principales exponentes fueron Pierre Massé y René Lenoir, quienes emplearon este concepto para aludir a los pobres y a "las circunstancias personales o grupales que impedían la integración a la sociedad bajo las mismas condiciones" (Padrón y Román, 2010: 810); también solía emplearse para "caracterizar a un grupo de ciudadanos marginados y desprotegidos que no recibían ningún tipo de prestación por parte del Estado" (Camarero, 1998, p. 76).

Durante las décadas del ochenta y noventa del siglo XX, el uso de este concepto de exclusión social reemplazó la noción de pobreza así como el debate sobre la redistribución de la riqueza; pasó a ser empleado no solo para referirse a la marginalidad y desprotección de algunas personas, con respecto a los sistemas universales de seguridad y asistencia social del Estado, sino también a la falta de vinculación e inserción social de muchas otras, debido, en su mayor parte, al desempleo o al empleo precario (Cubillos, 2017: 357). Desde finales de los noventa, a partir del proceso de globalización, con el predominio del neoliberalismo y el aumento de la desprotección social, se impuso una tendencia teórica que empleaba el término exclusión para entender "algo que va más allá de la pobreza y la desigualdad social, que se manifiesta en ruptura de los lazos sociales, dualismos y estratificación social" (Padrón y Román, 2010: 817); sobre esta perspectiva, la exclusión se relacionó, por una parte, con las condiciones de marginación y desprotección en las que vivian ciertas personas; mientras que, por otra parte, se asoció con una concepción de ejercicio de ciudadano (Camarero, 1998, p. 76).

En América Latina, el debate sobre la exclusión sucedió cuando en la región se discutían temas como la marginalidad, la desigualdad social, la pobreza y la constitución de las democracias modernas. El mayor predominio en el debate sobre estas últimas se debía a que las mismas se basan en la inclusión de sus ciudadanos para funcionar; desde este espacio de la discusión se entiende que "la exclusión ocurre dentro de los sistemas democráticos basados en la inclusión de sus ciudadanos en los distintos subsistemas sociales" (Padrón y Román, 2010: 817). Dada estas circunstancias, algunos autores sostienen que la inclusión social es un concepto más político que analítico, debido a sus efectos perjudiciales para la estabilidad de las democracias y, por el acceso desigual a derechos; por lo tanto, en el debate político, la desigualdad social se centra en la materialización de la inclusión (Muñoz y Barrantes, 2020: 13; Jelin, 2021:40). 
La categoría "inclusión" goza de un esfuerzo deliberativo dentro de los organismos internacionales, en su búsqueda por tratar de medirla y comprobar su efectividad. Desde esta perspectiva se entiende por inclusión a "un vector con un conjunto de dimensiones en el que los individuos son reconocidos y tienen garantía de acceso a derechos para desarrollarse en condiciones de igualdad" (CEPAL, 2017:11); a su vez, que "la desigualdad en el acceso a recursos, derechos, bienes y servicios, afecta la calidad de vida de las personas", así como su integración a la sociedad (Carrasco y Suárez, 2018:13). Esta línea posiciona el rol crucial, estructurante y productivo del Estado en términos de reconocimiento y protección de derechos; una mirada que se ubica en un espacio analítico predominantemente normativo en el que la inclusión es evaluada, o abordada, a través de los sistemas de protección social que surgen con el propósito, además de proteger de la pobreza, facilitar el acceso como garantía de inclusión, sobre la base del concepto y los principios de universalidad, no-discriminación e igualdad (Carrasco y Suárez, 2018:13; Cecchini y otros, 2015: 33; Muñoz y Barrantes, 2020: 18).

Desde otro punto de vista, el reconocimiento está más relacionado con una dimensión cultural, en el cual la inclusión significa pertenecer, ser incorporado, "adjuntarse"; es decir, se entiende como formas sociales de inclusión a las distintas conexiones interpersonales, al igual que aquellas en el marco de la sociedad de las que se desprenden leyes, reglamentos y normas que regulan el alcance de las libertades y las oportunidades de participación (Felder, 2018:302). Esta visión habla de la inclusión como formas reguladas de reconocimiento en las cuales la sociedad es considerada legítima por sus miembros, en la medida en que sea capaz de garantizar relaciones de reciprocidad y de reconocimiento en varios niveles (Felder, 2018: 310; Fraser, 2003: 22).

Para Thayer y Durán (2015) que basan su estudio en la teoría del reconocimiento expuesta por Honneth (2010), el reconocimiento se da en un contexto permeado por relaciones de poder entre sujetos culturalmente diferenciados (Thayer y Durán, 2015:140); esta propuesta conceptual afirma, además, que las políticas públicas con enfoque de derechos humanos como fundamento de la ciudadanía, segmentan a la población y tensionan su incorporación social. De acuerdo con estos autores, la desigualdad afecta de manera diferente a los distintos colectivos $\mathrm{y}$, además, se expresa en los altos niveles de vulnerabilidad que permean al fenómeno migratorio (Thayer y Durán, 2015).

Existen coincidencias teóricas que igualmente ubican a las desigualdades sociales y al debate entre inclusión versus migración como un vínculo en constante tensión, especialmente relevante en el ámbito del derecho y de las políticas públicas migratorias que hacen converger al Estado con el migrante dentro de un "hecho político", en el cual se deben su existencia el uno al otro (Oszlak y O’Donnell, 1981; Castles, 2006).

Hasta aquí se pueden demarcar al menos dos maneras de entender la inclusión a partir del reconocimiento. Una está relacionada con la conexión entre inclusión y reconocimiento en el marco de una reacción o respuesta social, o como un estatus atribuido, con la justicia y la manera en que los esquemas culturales institucionalizados impiden que los individuos participen en la vida social como iguales (Felder, 2018: 309). Esta postura agrega que las luchas por el reconocimiento tienen lugar en un mundo de desigualdades materiales y recorte de derechos (Thayer y Durán, 2015: 136) como el acceso al trabajo asalariado, la educación, la asistencia sanitaria y que "tiene consecuencias en las expectativas de vida y las tasas de enfermedad y mortalidad" (Fraser, 2003: 2).

La otra aborda el reconocimiento desde el aspecto normativo (García, 2016: 17), que, del mismo modo, impone un peso a la existencia de desigualdades; una precondición particularmente desafiante para la región latinoamericana, debido a su marcada desigualdad histórica y por las brechas u obstáculos para el acceso al derecho por parte de los sectores menos favorecidos (Muñoz y Barrantes, 2019:37). Estas dos visiones, lejos de ocupar espacios analíticos opuestos, se imbrican en la medida en que ambas están institucionalizadas en un Estado que crea el medio legal y regulatorio para el desarrollo de las estrategias sociales y al mismo tiempo, rompe con un núcleo común de protección en el que la población migrante se ubica como uno de los grupos más sensibles (García y Nejamkis, 2018:220).

Entonces, en el marco de discusión sobre la protección e inclusión social de la migración, se encuentran los debates sobre la desigualdad y 
pobreza, así como los que cuestionan la facultad que tiene el Estado para reconocer y garantizar los derechos, del mismo modo que para determinar quiénes son nacionales y organizar el reconocimiento de dichos derechos a partir de la clasificación entre nativos versus extranjeros (García, 2016: 120); en esta línea, y de manera similar, se ubican los análisis basados en la normativa mediante la cual se desarrollan e implementan las políticas migratorias como parte de los niveles diferenciales para la inclusión y el acceso a los servicios públicos y/o a los mecanismos de inclusión social (Ceriani, 2011).

Desde el campo del conocimiento sobre la visión normativa, se fragmenta el abordaje en dos dimensiones legales: la interna y la internacional. Esta perspectiva responde a la entrada de una nueva era de la migración que transformó las relaciones bilaterales y regionales, la seguridad, la identidad y la soberanía nacional, dado el encuentro con las lógicas regulatorias basadas en el paradigma de los derechos humanos. Específicamente, en América Latina esta nueva era está protagonizada por el crecimiento de la migración intrarregional que dio lugar a un marco conceptual sobre la "reciente humanización" en las legislaciones y políticas migratorias que, en Latinoamérica, permite hablar de un viraje en el inicio del siglo, impulsado por gobiernos progresistas que dirigieron esfuerzos hacia un entendimiento de la migración en clave de derechos humanos. Esta precondición posiciona a la persona migrante como sujeto de derechos humanos frente a un Estado del cual no son nacionales, pero, al mismo tiempo, conforma una relación ambivalente entre lo nacional, lo internacional y la persona migrante, cuando en estricto rigor es el Estado quien tiene la facultad de fijar sus políticas migratorias y de "asignar derechos distintos a nacionales frente a extranjeros, a migrantes en situación regular o irregular" (García, 2016:107; García y Nejamkis, 2018: 222; Nicolao,2019: 5).

A este respecto Domenech y Pereira (2017) destacan que el estudio que toma como objeto y foco de análisis las llamadas políticas migratorias, al menos desde las contribuciones más significativas durante los últimos 30 años, sigue monopolizado por el discurso académico sociojurídico, y se ha basado fundamentalmente en la legislación y programas nacionales de gobierno, atravesados por el nacionalismo metodológico y el pensamiento de Estado (Domenech y Pereira; 2017: 85), una perspectiva que soslaya la complejidad o las ambigüedades que convergen en el ámbito del gobierno de la migración, además que ha minimizado el proceso de producción estatal de la ilegalidad o irregularidad migratoria y ha bloqueado las formas de "problematizar la configuración histórica de los controles migratorios y fronterizos, y los orígenes de prácticas como la detención y la expulsión de extranjeros" (Domenech y Pereira; 2017: 99).

Aunque cada situación migratoria contiene sus propios desafíos de inclusión y de protección social, los estudios basados en la región latinoamericana la definen como un territorio fértil para la vulnerabilidad, la discriminación y la exclusión como consecuencia del origen, la raza, la condición sexual, entre otras características que interactúan con la categoría de extranjero y que podrían traducirse, no solo en prácticas discriminatorias, racistas y xenófobas, sino también barreras para el ejercicio de los derechos, principalmente el acceso a la protección social (Muñoz y Barrantes, 2020).

Visto hasta aquí, metodológicamente la normativa es la "puerta de entrada" para el estudio de las políticas públicas migratorias, pues se encuentran comprendidas en un cuerpo documental que constituye el conjunto de propuestas institucionales (leyes, decretos, resoluciones, directrices, acciones, $\mathrm{u}$ omisiones, etc.) que determinado Estado desarrolla sobre la entrada, salida y/o permanencia de la población nativa y/o extranjera dentro de su territorio (García y Nejamkis, 2018: 222; Novick, 2012:13; Nicolao, 2019: 6).

Es decir, las políticas públicas migratorias representan el modo de instrumentalizar el concepto de inclusión que en los criterios de legislación nacional se traducen en formas, condicionamientos y requisitos para la entrada o permanencia de los extranjeros. Un ejemplo tangible de cómo opera, está en la solicitud de documentos como los antecedentes penales o policiales, la defensa del empleo nacional, entre otros que forman un marco diferencial de ciudadanía definido a partir de niveles y categorías migratorias que dan el carácter de regularidad a partir del tipo de residencia: transitoria, temporal o permanente (García y Nejamkis, 2018: 233; Ceriani, 2011: 72; Domenech y Pereira; 2017: 96).

En un texto sobre Discursos y políticas de inmigración en Sudamérica, Acosta y Freier, (2015) tensionan el discurso político sudamericano 
sobre los derechos de los migrantes y la promoción de la movilidad humana con carácter universal. Este texto sostiene que si bien es cierto existe una apertura hacia un discurso universalista traducido en legislaciones de carácter innovador en algunos países, como por ejemplo en Argentina, se vislumbra al mismo tiempo una cierta preocupación a la hora de articular un discurso coherente respecto a las nuevas migraciones y la regulación legislativa dirigida específicamente a los migrantes en situación irregular, "ya que son los migrantes indocumentados los que tienen mayores dificultades a la hora de acceder a derechos y a un estatus jurídico seguro" (Acosta y Freier, 2015:174).

Emerge la regularidad migratoria como una variable que se define a través de un trámite administrativo resuelto en un tiempo que ubica a las personas migrantes al margen de la ley, expuestas a desprotección jurídica; es por esto que dentro de los determinantes que operan en el proceso migratorio -económicos, políticos, socioculturales, individuales y tecnológicos-, la irregularidad es un rasgo común para la profundización de la vulnerabilidad, en tanto que impone una limitación estructural en el acceso al bienestar, la protección social y al goce de los derechos humanos (Ceriani, 2011: 69; Penchaszadeh, 2012; Acosta y Freier, 2015: 174; García y Nejamkis, 2018:226; Nicolao, 2019:17); dicha limitación comienza por la tensión que genera la identidad jurídica como el mínimo umbral de ciudadanía orientado a ciudadanos y residentes legales (Thayer y Stang, 2017).

Con base en la forma y el cómo los Estados configuran las trayectorias migratorias hacia el acceso creciente o decreciente a derechos, la irregularidad migratoria está asociada con los estudios sobre la desprotección social, explotación de personas, trabajo forzado, trata, prostitución y secuestro, como parte de algunos de los peligros que pueden manifestarse durante el trayecto migratorio. Por un lado, la falta de documentos en el país de acogida posibilita la ilegalidad que conduce a procesos judiciales o administrativos como multas, deportación, etc.; por el otro, no contar con la documentación del país de origen (partidas de nacimiento, comprobantes de matrimonio y otros), igualmente aumenta el riesgo de caer en redes de abuso o de utilización de canales poco convencionales para acceder al trabajo, la justicia y los servicios públicos. Queda así descrita la vulnerabilidad migratoria, en un tránsito que interpela el peso determinante del Estado, su centralidad y, por ende, la creciente necesidad de abordajes, tratamientos y políticas públicas internacionales con base en la protección jurídica dentro de un sistema supranacional (Nicolao, 2019; Thayer y Stang, 2017).

Para sistematizar las ideas expuestas hasta ahora, la migración es un hecho social que converge en los márgenes de discusión entre los ámbitos global, internacional, regional, nacional y local, y en su estudio existe una tendencia que privilegia el abordaje desde los marcos normativos, como modo de garantizar la inclusión y el acceso por ley a la protección social, lo que deja implícita una concepción o tutela estatal que se define por medio del conjunto de derechos civiles, políticos y socioeconómicos que tienen las personas migrantes para insertarse o integrarse en la sociedad de destino (Gíl y Santi 2019:5; Thayer y Stang, 2017).

Paralelamente, con el crecimiento de la movilización transnacional tomaron fuerza en las investigaciones los instrumentos normativos internacionales a los que se adhieren los Estados, como referencias para el análisis de la temática de inclusión de la migración, así como de la implementación del enfoque de derechos humanos en los sistemas nacionales de protección social; pues "su incorporación en las normas nacionales constituye, al menos desde el ámbito jurídico, un respaldo para el acceso a derechos" (Novick, 2012: 13; Domenech y Pereira; 2017: 95; García y Nejamkis, 2018: 232; Gíl y Santi, 2019:6).

La instrumentalización de la norma internacional se define a través de un conjunto de intervenciones que el Estado realiza en la esfera pública, asumiendo la provisión de los denominados servicios universales (Canelo, Gavazzo y Nejamkis: 2018). Al respecto Nicolao (2019) señala que "si bien es cierto que los movimientos migratorios internacionales poseen una dimensión global, es en el ámbito local donde se delimita" (Nicolao, 2019: 4), lo que significa que en definitiva, aunque los Estados han ratificado un conjunto de tratados internacionales y regionales en el plano de la gobernanza global de las migraciones, y además, en el Derecho Internacional han iniciado un recorrido en materia de respeto, garantía y protección de los derechos humanos de las personas migrantes, las políticas migratorias son elaboradas y ejecutadas a nivel nacional (Nicolao, 2019; García y Nejamkis, 2018). 
Quizás esta discrecionalidad se debe a que dentro de las principales respuestas que acompañan a la inmigración contemporánea, “en el marco de nuevas (viejas) políticas migratorias prevalece un contexto de rupturas y continuidades", en un debate constante sobre la criminalización de la migración, la persistencia de componentes securitarios y desigualdades múltiples (Canelo, Gavazzo y Nejamkis, 2018:155; Jelin, 2021:41). En el marco de esta discusión, en torno a la necesidad y preocupación subyacente, este estudio se inserta en la tensión entre la norma y su aplicabilidad, en el marco de la gobernanza de la migración basada en los derechos humanos y en los diversos instrumentos internacionales que acompañan la inclusión social y el acceso a la salud de la población migrante.

\section{Marco jurídico de protección e inclusión social al migrante}

Más allá del hecho de ser nacional de un Estado, el concepto de inclusión se imbrica con las premisas de protección universal, definidas como el complemento al derecho interno de los Estados que reafirman instrumentos tanto en el ámbito internacional como en el regional. Visto de esta forma, se podría decir que la gobernanza de la migración internacional opera desde dos ámbitos: en el Sistema de Protección Universal y el Sistema Interamericano, cada uno de ellos dispone de diferentes instancias, mecanismos y organismos destinados a la protección y garantía de los derechos humanos en los distintos niveles de acción.

A nivel internacional, existen un conjunto de instrumentos jurídicos vinculantes $y$ otros conexos, junto con normas no vinculantes. Dentro de los instrumentos específicos consagrados en el marco de los derechos humanos para la protección de los migrantes, destaca por su carácter vinculante la Convención Internacional sobre la Protección de Derechos de Todos los Trabajadores Migratorios y de sus Familiares, de 1990 (ONU, 2011). Por otra parte, en el Sistema Interamericano de Derechos Humanos se puede observar la Convención Americana sobre Derechos Humanos, conocida como "Pacto de San José" (1969), que definen la garantía de los derechos a toda persona sin discriminación (art 1.1); el derecho a la residencia (art 22), a la nacionalidad (art 20) y a la igualdad ante la ley (art 24); el Protocolo Adicional a esta
Convención Americana, específicamente en materia de Derechos Económicos, Sociales y Culturales conocido como "Protocolo de San Salvador" (1988), que establece la obligación de los Estados de adoptar medidas (art 1) y disposiciones del derecho interno (art 2) (Jaramillo,2013: 55).

Dentro del ámbito intergubernamental, también se pueden identificar esfuerzos dirigidos a promover instancias de cooperación y discusión en torno a la migración y la protección del migrante. Un ejemplo es el Pacto Mundial para una migración segura, ordenada y regular (2018), que es un documento esencial para analizar lo relativo con la gobernanza migratoria y la garantía de derechos por parte de esta población. Bajo este panorama, el acceso a los servicios sociales es clave para el estudio de la inclusión social del inmigrante, ya que la garantía de las necesidades básicas y el acceso a servicios como salud, educación y trabajo son fundamentales para garantizarla. Ante este desafío, en septiembre de 2015 la ONU adoptó la Agenda 2030, que buscó dar impulso a los acuerdos $\mathrm{y}$ compromisos asumidos a nivel bilateral $\mathrm{y}$ multilateral, con énfasis en la estrecha relación entre migración internacional y la protección social de las personas migrantes. Además, desde el año 2016 y 2017 la Asamblea General de las Naciones Unidas activó las negociaciones en torno al Pacto Global de Migración. En América Latina, se instaló en la idea de protección universal y sus lineamientos políticos bajo la perspectiva de desarrollo humano para las migraciones; ante dicha perspectiva, se planteó la construcción del Plan Sudamericano de Desarrollo Humano de las Migraciones (PSDHM-2010).

Por su parte, el carácter multilateral de la gobernanza migratoria se expresa en distintas respuestas regionales a nivel mundial, y es a través de la plataforma del multilateralismo que se han formulado políticas específicas para facilitar la circulación de personas en cada región, con cierto grado de concesión de soberanía a un nivel de autoridad más elevado, como es el caso de las comunidades políticas y económicas regionales (Acosta y Freier, 2015). Por ejemplo, en torno al caso del Mercado Común Latinoamericano (Mercosur), bajo el pragmatismo económico y en torno al área de libre comercio, se instaló una línea entre los derechos humanos y la regularización de migrantes; en lo específico, se impulsó el Acuerdo sobre Residencia para Nacionales de los Estados Parte, como un modo de facilitar la movilidad, la 
residencia y la regularidad de las personas en este espacio geográfico (Brumat, 2019:11). Algunas opiniones señalan que este Acuerdo se convirtió en "un salto cualitativo fundamental en el tratamiento dado a la movilidad territorial" (Novick, 2011:21; Cerani, 2011:78); de hecho, desde que se firmó en el 2001, no ha existido otro documento de relevancia regional con tanto impacto sobre los derechos de los migrantes, especificamente para regularizarse y adquirir la residencia (Ramírez, 2019: 32).

En sinergia con el enfoque de Derechos Humanos adoptado por el Mercosur y en términos de normativas, algunos de los países de la región se enrumbaron hacia la discusión y aprobación de nuevas leyes migratorias (Brumat, 2019: 12). En este sentido, Argentina (2003) fue el primero en avanzar con la sanción de una legislación que reconoce a la migración como un derecho humano (Canelo, Gavazzo y Nejamkis: 2018:154). Sin embargo, algunos estudios sostienen que la normativa y el discurso sobre la migración como derecho humano en América Latina aún coexiste con el enfoque de seguridad (Domenech y Pereira, 2017:95; Gil Araujo y Santi, 2019).

Esto quiere decir que, aunque la agenda regional avanzó en la discusión sobre los derechos humanos de las personas migrantes y, en algunos casos se tradujo en la reconfiguración del marco normativo legal, como el caso de Argentina que pasó a identificarse como una normativa de avanzada en la materia, el debate sobre la inclusión en términos de acceso a los derechos sociales por parte de los migrantes aun presenta serios desafíos relacionados sobre todo con la regularidad migratoria, en tanto el vínculo entre migración y ciudadanía (Thayer y Stang, 2017; Novick, 2011; Basualdo, 2017).

En el ámbito de lo nacional, con la sanción de la Ley de Migraciones № 25.871 (2004) Argentina "parte de reconocer a la migración como un derecho fundamental e inalienable de la persona, que se debe garantizar sobre la base de la igualdad de trato" (Nicolao, 2019: 6). En este sentido, lo primero que dice sobre su política migratoria, es que se encuentra explícitamente contenida en normas, leyes, decretos, resoluciones, tratados internacionales y regionales que conforman el sistema universal e interamericano de derechos humanos. En cuanto a la regulación, a través de la Ley № 25.871, 2004, art. 23, inciso 1 , incorpora la categoría de "residencia temporaria por nacionalidad" que establece el derecho a una residencia por dos años, prorrogables con entradas y salidas múltiples a los ciudadanos provenientes de países miembros y asociados del Mercosur (Nicolao, 2019: 7).

Con base en el modelo regional, Argentina postula una Ley considerada de avanzada por algunos organismos internacionales, dado a que establece la migración como un derecho humano e incorpora la igualdad en el acceso y protección de derechos sociales de las personas migrantes.

\section{Inclusión de la migración a la salud pública en Argentina}

En este campo de discusión sobre la protección e inclusión de la migración a los derechos sociales, el tema de la salud ha sido especialmente relevante al interior de la ONU; de hecho se creó el concepto vinculado con la salud global para operacionalizar su relación con los derechos humanos y la política intergubernamental, el mismo establece que "el goce del grado máximo de salud que se pueda lograr es uno de los derechos fundamentales de todo ser humano sin distinción de raza, religión, ideología política o condición económica o social" (OMS, 1946, 2015: Preámbulo), un concepto íntimamente ligado con "el proceso histórico de la globalización económica y tecnológica y a la emergencia de problemas planetarios relacionados al medio ambiente y a los nuevos flujos migratorios" (Manchola, Garrafa, Cunha y Hellmann, 2017: 2152).

Además, en los distintos abordajes es frecuente advertir que las personas que migran muestran una importante vulnerabilidad en su salud, debido a los desajustes emocionales que supone dejar el lugar de origen, los cambios de alimentación o las dificultades para alimentarse adecuadamente, la incertidumbre de la travesía entre otras circunstancias (Piñones, Liberona, Nanette y Leiva, 2021).

En consecuencia, se han creado mecanismos internacionales que están relacionados con el derecho a la salud para la protección de las personas migrantes. Tal es el caso de la resolución de la OMS $\mathrm{N}^{\circ}$ WHA61.17 de Salud de migrantes del 2008, la Declaración del Diálogo de Alto Nivel sobre la Migración Internacional y el Desarrollo de octubre de 2013 (Resolución A/RES/68/4) de la ONU, el marco operación de referencia Consulta Global en Salud de Migrantes de Madrid, basada 
en la resolución WHA61.7 de 2010, y el Informe de Promoción de Salud de Migrantes de la OMS (A69/27) de 2016, la resolución CD55/11 de OPS la Salud de los Migrantes de 2016, la Declaración de Colombo de la Reunión Global sobre Salud de los Migrantes, de OIM y OMS de 2017.

Sobre la base del marco migratorio garantista en materia de salud a nivel global, la ley argentina señala que "no podrá negársele o restringírsele en ningún caso, el acceso al derecho a la salud, la asistencia social o atención sanitaria a todos los extranjeros que lo requieran, cualquiera sea su situación migratoria” (Ley № 25.871, 2004, art. 8).

Ahora bien, algunos estudios que reflexionan sobre el binomio migración y salud en Argentina sostienen que se trata de un sistema de protección social desigual, con presencia de "mecanismos de inclusión diferencial" que regulan el acceso de la población no nacional "cuando las prácticas que demandan son costosas" (Nicolao, 2019: 6; Basualdo, 2017: 68). Dichos estudios basan sus análisis en la aplicación de la norma, el funcionamiento de la institucionalidad en las distintas esferas de atención y el contraste entre la garantía de inclusión y la demanda de bienes y servicios; además, cuestionan la universalidad y el acceso reducido para los migrantes, sobre todo en situación "irregular" (Goldberg y Silveira, 2013; Nicolao, 2019; Basualdo, 2017).

En la bibliografía consultada se desprenden al menos tres formas de abordaje que, en su mayoría, toman como referencia los migrantes bolivianos, paraguayos y peruanos en contextos locales (Courtis et al., 2010; Goldberg y Silveira, 2013). La primera está relacionada con los determinantes sociales en la salud (DSS); la segunda, desde las desigualdades y los aspectos diferenciales que imponen la cobertura y la utilización de los servicios como brechas para el acceso al servicio; la tercera, asociada con el enfoque de derechos humanos desde la perspectiva de la salud pública.

Los determinantes sociales de la salud son aquellas condiciones en las que las personas nacen, crecen, viven, trabajan y envejecen y que, a su vez, determinan la concepción de los sistemas de salud; dichos determinantes generalmente se relacionan con la distribución de la riqueza, el poder y los recursos a nivel mundial, nacional y local, y con los sistemas políticos adoptados por los países (CEPAL, 2009; OMS, 2008). Existen otros determinantes sociales que actúan como brechas para el acceso a la salud como un derecho, y son los relacionados con las condiciones de exclusión, fragmentación y discriminación que incrementan la vulnerabilidad de los migrantes, como consecuencia del estigma y las barreras culturales y/o comunicativas (Mario, 2018).

$\mathrm{Si}$ bien es cierto que la migración en general es considerada un determinante social, particularmente la irregular funciona como determinante social de la salud; aparece nuevamente la variable sobre la regularidad migratoria, como uno de los principales obstáculos para el acceso a derechos como la salud, ya que está sujeto a las condiciones específicas en las que se realiza la migración, es decir, si es voluntaria o forzada, si es en contexto de frontera, la etnicidad, el género, el país de origen y de destino, puede aumentar el riesgo de afectar la salud de las personas (Goldberg y Silveira, 2013; Nicolao, 2019). Desde otro punto de vista, Piñones, Liberona, Nanette y Leiva (2021) señalan que una de las aproximaciones contemporáneas más vigorosas en el abordaje del binomio saludmigración es el de la vulnerabilidad estructural que, a diferencia de los DSS, muestra cómo la estructura social impone riesgos y constricciones específicas a la salud individual y colectiva. Sostienen que para abordar el tema de la salud de los migrantes se debe considerar, en primer lugar, el problema de la estructura económica y política, o el modo de producción, es decir, la vulnerabilidad estructural como herramienta para comprender cómo los mecanismos de la violencia simbólica, presentes en la naturalización de las jerarquías sociales y el racismo estructural, son materializados en los procesos de irregularización administrativa y la producción de entornos sociopolíticos iatrogénicos (Piñones, Liberona, Nanette y Leiva, 2021; Cabieses y Oyarte, 2019).

En consonancia con lo anterior, una segunda forma de abordaje sobre migración y salud encuentra su objeto de análisis en el campo de las desigualdades que funcionan como las brechas para el acceso al servicio; todo lo cual se enmarca en el tópico la protección social y en las políticas de salud que se suman a las intervenciones que el Estado realiza asumiendo -o no- la provisión de los servicios universales (Molina, 2019). En el marco de esta lógica, se define la "situación de vulnerabilidad en salud", un concepto empleado para aludir a la desprotección de ciertos grupos poblacionales ante 
daños potenciales específicamente a su salud, como consecuencia de los obstáculos y desventajas que subyacen frente a cualquier problema de salud (Piñones, Liberona, Nanette y Leiva, 2021).

Ballesteros (2014) sostiene que las desigualdades en el acceso a la salud y al sistema sanitario están condicionadas fundamentalmente por la clase social y otros anclajes estructurales de los sujetos (género y grupo etario), así como con el ingreso, el tipo de cobertura de salud y el lugar de residencia; a partir del enfoque teórico de Andersen (1995), la autora sostiene que, para comprender las desigualdades en el acceso a los servicios de salud es necesario examinar las necesidades diferenciales de utilización de dichos servicios (Ballesteros; 2014). En este mismo sentido Mario, S. (2018) señala que las situaciones de desigualdad, así como las de exclusión social, están demarcadas por la distribución de los ingresos y de las condiciones materiales de vida, y que éstos son los principales obstáculos para el acceso efectivo a los servicios de atención a la salud. El mismo autor sostiene que las desigualdades en la salud también se observan a partir de indicadores sobre los distintos sistemas de cobertura y mediante las prácticas preventivas relacionadas con la percepción del estado de salud (Mario, 2018).

La bibliografía que identifica la existencia de obstáculos en la provisión y la utilización de los servicios de la salud en el caso de Argentina, indica que a raíz de los procesos de descentralización del financiamiento y de la gestión de los servicios de salud se puso en relieve, por un lado, que la provisión depende de las capacidades financieras y de los recursos disponibles a nivel local, es decir, las diferencias en el tipo de cobertura de salud están condicionadas por los niveles de ingresos $u$ ocupación de las personas; y por el otro, la utilización de los servicios está más sujeta a cuestiones biológicas, -como el padecer una enfermedad-, que están mediadas por la edad, el género, las creencias y prácticas en torno al cuidado del propio cuerpo, entre otros (Nicolao, 2019; Mario, 2018).

Ballesteros y Freidin (2009) definen, por un lado, el acceso al sistema de salud entendido como la previsión de salud; y por el otro, el uso efectivo de servicios de salud, desagregado en necesidad sentida, consulta o cobertura, barreras y satisfacción de la necesidad. Estos autores ubican dentro de las barreras para el acceso, indicadores que impiden o dificultan la obtención de atención una vez detectada la necesidad, como, por ejemplo, la disponibilidad del servicio, los tiempos de espera, la distancia y los costos (Ballesteros y Freidin, 2015). Otras visiones abordan el acceso a través de la utilización de los servicios en un período determinado, la accesibilidad, las necesidades de salud, los factores demográficos y la presencia de recursos que posibilitan el uso de los servicios, algunas veces encuentran que la consulta con el médico durante el último año es el indicador más utilizado para medir el acceso a los servicios de salud (Cabieses y Oyarte, 2019; Molina, 2019).

Una tercera visión sobre el acceso y la utilización de los servicios se encuentra en la cobertura universal y el enfoque de los derechos humanos dentro de la perspectiva de salud pública, en tanto acceso equitativo a la atención, más allá de la condición migratoria de las personas (Manchola, Garrafa, Cunha y Hellmann, 2017:215; Cabieses y Oyarte, 2019). Dicho enfoque posiciona la salud como un derecho universal inalienable y la protección social en salud representa una garantía de ese derecho. Según la OPS esta protección se desarrolla a través de tres dimensiones principales y complementarias: cobertura horizontal (acceso al sistema de salud); cobertura vertical (acceso a prestaciones) y protección financiera. La primera se encuentra vinculada con el indicador de acceso al sistema de salud y el uso o utilización efectiva del servicio o prestaciones de salud (OPS, 2010).

Esta discusión sostiene que "el hecho de clasificar a la salud como un derecho humano es uno de los aspectos más polémicos dentro del área de la salud global" porque "involucra costos y temas relativos a la importante injusticia e inequidad sanitaria que una significativa porción de la población enfrenta por estar inmersa un sistema neoliberal injusto que excluye a millones de personas" (Manchola, Garrafa, Cunha y Hellmann, 2017: 2153). Lo quiere decir es que asumirla como un derecho humano tiene implicaciones profundas en el escenario internacional, no solo porque hace que los Estados asuman obligaciones legales frente a la comunidad internacional, sino porque su garantía también está asociada con grandes costos en los sistemas nacionales dirigidos a su atención (Manchola, Garrafa, Cunha y Hellmann, 2017:2153).

$\mathrm{Si}$ bien es cierto que tradicionalmente "la agenda política pública no encuentra en la temática de la atención a la salud un objetivo 
prioritario" (Maceira, 2020: 156) no es menos cierto que "el incremento de los costos que trae consigo la incorporación de nuevas tecnologías, la identificación de derechos con el compromiso de garantizar espacios de cobertura y el debate internacional del acceso universal" vislumbran la necesidad de profundización de este debate sobre la salud dentro de la agenda de política pública (Maceira, 2020:157). En ese sentido, Molina (2019) argumenta que en Argentina el ejercicio del derecho a la salud de la población migrante está ligado a debates y tensiones más generales en torno al alcance de los servicios, el modelo de salud pública, de atención dominante y las nociones de accesibilidad que orientan la política sanitaria junto con las prácticas de los equipos de salud; desde su perspectiva, la accesibilidad es un vínculo a construir entre los sujetos y los servicios (Molina, 2019).

En sintesis, sobre la base de un panorama de garantía para el acceso, la inclusión del migrante a la salud argentina se define como "la asistencia gratuita, efectiva en lo sanitario y receptiva desde el punto de vista cultural" (Molina, 2019: 5). Bajo este postulado, se parte de la relación del sistema de salud público nacional y local argentino, en clave con la lógica de salud como un derecho humano (Nicolao, 2019); derecho muchas veces reducido ante la presencia de mecanismos de inclusión diferenciada que están relacionados "con la preocupación por resguardar los bienes sociales proporcionados por el Estado para los nacionales o extranjeros-residentes, incluido el acceso a ciertos tratamientos de salud" (Basualdo, 2017:69; Goldberg y Silveira, 2013:287).

En cualquier caso, abordar el sistema de salud exige el diálogo constante con la existencia del derecho universal a la protección de la salud, entender que se trata de un debate más amplio, sujeto a tensiones generales relacionadas con las nociones de accesibilidad, las prácticas de los equipos de salud, entre otras discusiones (Molina, 2019); también supone no perder de vista que, más allá de la cobertura que brinda el Estado, existe la premisa de que las personas migrantes inicialmente no cuentan con cobertura médica.

\section{El sistema de salud pública argentino}

Según la OMS, el sistema de salud representa las distintas formas que adoptan la protección de los riesgos y los distintos daños a la salud; este organismo entiende a la salud pública como un estado de completo bienestar físico, mental y social, y no solamente la ausencia de afecciones o enfermedades (OMS,1948). En palabras de Mannoukian y Venturini, citando a Dever (1991) "la salud no es solamente el producto de condiciones genéticas, biológicas y emocionales, sino también el resultado de relaciones sociales predominantes, por la estructura y dinámica de la sociedad"; además, "determinada por el momento histórico que le enmarca, las relaciones políticas y la organización del Estado” (Mannoukian y Venturini, 2021: 43). Aunque estos conceptos han presentado ajustes según el momento de la historia, a partir de la década de 1990, la estrategia internacional sobre la salud se basó en la promoción de su garantía como acceso universal (Mannoukian y Venturini, 2021).

Ahora bien, la literatura que registra la huella de esta década en el sector de la salud, también reporta un inicio de siglo marcado por los estragos del neoliberalismo y su impacto negativo sobre los sectores menos favorecidos como los inmigrantes, los pueblos originarios y, en definitiva, los excluidos de un sistema pensado para pocos (clase-etnia-género). Dicha realidad dio lugar a la multiplicación de los estudios y las denuncias sobre las desigualdades sociales, en salud y sobre las brechas que se intensificaban entre naciones y dentro de un mismo país. Aunque aún se disputa la implementación de un concepto amplio para explicar estas brechas u obstáculos; por un lado, el de la OMS y el sistema internacional que postulan la presencia de los determinantes sociales y, por el otro, la prevalencia de procesos estructurales que provocan y promueven la desigualdad, existe una marcada coincidencia en los puntos de vista abordados de que, al menos en Latinoamérica, la salud va más allá de los determinantes sociales y que la desigualdad es, más bien, el telón de fondo de donde emergen (Mannoukian y Venturini, 2021).

Mannoukian y Venturini (2021), describen cuatro dimensiones para analizar la salud como sector: el estado de salud, las políticas de salud, los sistemas de salud y los sistemas de atención médica. El estado de salud está relacionado con el punto del que se desprenden las políticas dirigidas a este sector; tiene que ver con el diagnóstico de la situación epidemiológica de los integrantes, el abordaje sobre la enfermedad y la muerte, entre otras cuestiones sociales sobre la salud colectiva 
(Mannoukian y Venturini, 2021). Por su parte, la OMS define a los sistemas de salud como la suma de organizaciones, instituciones y recursos cuyo fin último es mejorar la salud (OMS, 2005). Mientras que los sistemas de atención médica son la fracción del sistema de salud que desarrolla acciones, actividades, tareas y mecanismos puestos a disposición de la atención sanitaria, es el punto donde se indaga sobre los recursos destinados a la atención de las prioridades definidas (Mannoukian y Venturini, 2021).

En el caso argentino, se habla de un sistema fragmentado con diferentes formatos que conviven en un mismo territorio y abastece a distintos grupos poblacionales de la misma localidad o región. Es quizás uno de los más fragmentados y segmentados de la región; está compuesto por tres sectores: el público, el de la seguridad social y el privado. El término de segmentación está relacionado principalmente con las fuentes de financiamiento que se extienden desde los impuestos nacionales, provinciales y municipales, hasta las contribuciones de la seguridad social (Maceira, 2020).

En líneas generales, por tratarse de un país federal cada una de las 24 jurisdicciones retiene su responsabilidad constitucional para ejercer con autonomía las funciones de rectoría, financiamiento y prestación de los servicios de salud, y en el cual la seguridad social se desarrolló históricamente de manera desintegrada con la mayoría de las entidades. Se conforma por el ministerio de la nación, los ministerios de salud provinciales, la red de hospitales y los centros de salud públicos, responsables de prestar atención gratuita a toda persona que lo demande, fundamentalmente a personas de los quintiles de ingreso más bajos, sin seguridad social y sin capacidad de pago. Este sector se financia con recursos fiscales ascendentes a $2.2 \%$ del PIB (que provienen fundamentalmente de aportes provinciales) y recibe pagos ocasionales del sistema de seguridad social cuando atiende a sus afiliados (Mannoukian y Venturini, 2021).

Dado que se habla de un sistema de salud caracterizado por su excesiva fragmentación, hay que considerar que esto también se expresa en las distintas fuentes de financiamiento, diferentes coberturas, coseguros, copagos aplicados y diferentes regímenes, órganos de control y fiscalización; es decir, tiene una estructura mixta y descentralizada sometido a normativas emanadas desde las distintas jurisdicciones. Se sustenta sobre mecanismos de protección financiera fuertemente condicionados por el sistema laboral, anclado a un esquema de protección institucional que descansa en espacios públicos de cobertura universal financiados por impuestos o a través de estructuras de seguro social que descansan en las retenciones en el trabajo. El sector público es el responsable de las necesidades de atención de salud de la población sin cobertura (Mannoukian y Venturini, 2021).

Con respecto a los abordajes sobre el sistema de salud y el acceso de la migración, es común observar que se basan en el relevamiento de datos provenientes de censos y encuestas puntuales a través de las cuales discuten la situación de la población y se visualizan en qué medida la condición de inmigrante es el principal factor diferenciador, como grupo social más desprotegido social y económicamente (Maceira, 2020; Molina, 2019; Mannoukian y Venturini, 2021).

Igualmente, existen análisis que privilegian el debate sobre la inclusión de la migración al sistema de salud argentino e identifican brechas existentes para postular una inclusión diferenciada en el acceso (Nicolao,2019; Basualdo, 2017; Manchola, Garrafa, Cunha y Hellmann, 2017; Cabieses y Oyarte, 2019; Molina, 2019; Mario, 2018; Ballesteros; 2014; Piñones, Liberona, Nanette y Leiva, 2021). Asimismo, se puede observar una tendencia de pensamiento que se instala en la lógica normativa, sobre la protección que precisan el objeto de un derecho, es decir, lo que de algún modo se define a través de la inclusión en las políticas y se garantiza, respalda u obliga por ley, con poca extensión y profundidad en torno a la tensión que conllevan las lógicas del país de origen en los centros de atención a la salud.

En cuanto a la caracterización demográfica, los diferentes estudios indican que mayoritariamente la población extranjera se concentra en el Gran Buenos Aires GBA, región que hoy absorbe al $62,3 \%$ de los inmigrantes, pero al mismo tiempo, se trata de una composición variada de acuerdo a cada provincia. Por un lado, la población migrante histórica tiende a radicarse en provincias fronterizas con su país; mientras que los nuevos contingentes de personas migrantes muestran una clara preferencia por la Ciudad Autónoma de Buenos Aires CABA (Mario, 2018). Tal es el caso de las personas venezolanas y colombianas a quienes se aplica el Acuerdo de Residencia para los Nacionales de los Estados Parte y Asociados del Mercosur, permiso de residencia (temporal y permanente). 
De acuerdo con esta información, sustentada por la encuesta de hogares de la ciudad, la distribución reciente corrobora el peso de los inmigrantes que comienzan a residir en la CABA en los últimos cinco años (Martínez y Carpinetti, 2021). De hecho, en un informe publicado en el año 2017 , el Mercosur alertó sobre la necesidad de dirigir la mirada de la inclusión social de los migrantes, hacia análisis cada vez más focalizados en las ciudades, los municipios y las áreas metropolitanas, es decir, en la actualidad las ciudades constituyen un espacio privilegiado para el estudio sobre la inclusión social de la población migrante (Nicolao, 2019).

Sobre la inclusión de la migración a la salud pública desde este ámbito local, la Ley Básica de Salud 153 de la CABA indica que razones de procedencia, nacionalidad, religión, condición social, migratoria, de enfermedad u otras no pueden conformar una barrera en el acceso a la atención de la salud. También reconoce que la gratuidad es condición necesaria e indispensable para garantizar este derecho, por ello explicita que ninguna forma de pago directo (cuota, bono de cooperadora) debe estar asociada a la prestación pública de salud.

Esto quiere decir que, aunque los servicios públicos de salud tienen por mandato constitucional la misión de asegurar el acceso igualitario y la Ley de Migraciones aprobada en 2010 señala que "no podrá negársele o restringírsele en ningún caso el acceso al derecho a la salud, la asistencia social o atención sanitaria a todos los extranjeros que lo requieran, cualquiera sea su situación migratoria" (art. 8 de la Ley 25.871), "la escasez de recursos coloca en el centro del debate la cuestión de cómo distribuirlos y quienes deben tener prioridad" (Jelin, 2011:7).

Hay que señalar que, aunque la ley extiende la cobertura de derechos sociales de los migrantes hacia todas sus jurisdicciones, nacional, provincial y municipal (Ley № 25.871, 2004: art. 6, 7. 14), la evidencia detenta una esfera de vaguedad en la distribución de responsabilidades y competencias (Nicolao, 2019).

De hecho, en su estudio Nicolao (2019) habla sobre los desafios en el acceso a los derechos sociales por parte de la población migrante, e indica que uno de los nudos críticos para el acceso a la salud es la existencia de discursos o prácticas discriminatorias por parte de profesionales de la salud, o de sus pares nativos en contextos de atención médica, desde las cuales se visualiza a los migrantes como abusadores de los servicios sociales y de salud pública, este estudio toma el caso de los inmigrantes en Bahía Blanca (Nicolao, 2019).

Con este mismo tenor, Molina (2019) reporta una investigación sobre violencia institucional, en la que registra un conjunto de conflictos vinculados con la atención médica de personas procedentes de Bolivia, Perú y Haití, y discute sobre la discrecionalidad de la Ley y el posicionamiento de un discurso xenófobo en el cual se culpabiliza a las personas migrantes por la pauperización y sobresaturación del sistema de salud; esta violencia fue desencadenada por funcionarios del Ministerio de Salud de Mendoza (Molina, 2019).

Lo visto hasta aquí no es una pretensión de simplificar el debate, ni mucho menos ser exhaustivo con unos resultados que distan de ser definitivos. Más bien, el objetivo es presentar, grosso modo, el camino que ya algunos autores han vislumbrado, desde la ley o convenio hasta su reglamentación, y la brecha entre la norma, la reglamentación y la implementación de esta; incorporar la idea sobre los marcos normativos de protección a los derechos humanos que coexisten con ambigüedades que tensionan su aplicación efectiva.

Existe basta literatura que demuestra los altos niveles de vulnerabilidad en torno a este fenómeno; no obstante, aún falta mucho por profundizar y actualizar en torno a inclusión, migración y salud, estudios basados en el nuevo mapa migratorio argentino, muy abordado desde los organismos internacionales, pero con amplios espacios teóricos y académicos por ocupar.

\section{A modo de cierre}

En América Latina, el debate entre inclusión social y protección a la migración aparece vinculado con la tradición académica del estudio sobre el Estado, la pobreza y la desigualdad latinoamericana; el mismo que en un contexto globalizado instaló su atención en el estudio sobre la problematización de la migración, considerada y conceptualizada como un problema social, económico, político y cultural para los Estados y las sociedades destino. Sin embargo, existe un conjunto de disposiciones que contemplan la inclusión a derechos de la población migrante, con ellas es notable un progresivo cuestionamiento al corpus de garantías que establece la normativa en sus distintos niveles, internacional, regional y nacional. 
En el contexto nacional argentino los derechos de los extranjeros están amparados por la Ley Nacional de Política Migratoria 25.871, que reconoce a la migración como un derecho humano que el Estado debe proveer en condiciones de igualdad con respecto a los nacionales y de universalidad. De tal modo que por Ley se garantiza el acceso igualitario a los servicios públicos como salud y seguridad social y sobre base de dicha Ley, los servicios públicos tienen el deber de atender a todas las personas sin distingo alguno.

No obstante, la evidencia explorada indica que cotidianamente se producen situaciones que reducen el alcance de dichos derechos, "en especial cuando los demandantes provienen de países pobres" (Molina, 2019: 2). En otras palabras, la Ley migratoria también ha sido marcada por un contexto discriminatorio, con registros de actos $\mathrm{u}$ omisiones por motivos de etnia, nacionalidad (entre otros), que arbitrariamente impiden, obstruyen o restringen el pleno ejercicio sobre bases igualitarias de los derechos y garantías fundamentales reconocidas.

En lo que respecta al acceso al derecho a la salud, el marco normativo en los diferentes niveles: supranacional, nacional y local, lo reconocen como un derecho humano básico y, al mismo tiempo, una obligación indelegable del Estado (sea nacional, provincial o municipal) que además tiene la responsabilidad de garantizar su provisión a todas las personas, sin restricción alguna.

Por su parte, la salud en Argentina se trata a través de tres sectores: público, de seguridad social y privado; debido a su fragmentación, según el reporte del Censo 2010, el 36\% de la población no tiene cobertura médica, lo cual indica que el debate sobre el porcentaje de personas que se atienden en hospitales y centros de salud públicos en todo el país, esconde importantes desigualdades, ya que hay grandes diferencias entre las provincias. Esta realidad ha sido descrita en muchos de los abordajes sobre inclusión de la migración a la salud pública en Argentina, en la que la exclusión se manifiesta en un discurso sobre la optimización o saturación de recursos sanitarios, a través del cual se "justifica" la implementación de mecanismos de inclusión diferencial. Dentro del panorama analítico se registran inequidades en los resultados en salud, en el nivel de gasto, así como en las condiciones humanas y materiales para el acceso efectivo a los servicios en los diversos territorios y grupos poblacionales de la nación.
En el área de la salud, aunque aplicable a todos los ámbitos sociales, subyace la violencia simbólica que habitúa las desigualdades sociales y actúa como el principal determinante social para la salud. Es importante decir que, en el marco del derecho, la garantía en sí misma no está vinculada con el goce de este. En todo caso, para los estudiosos de la materia, su definición implica una ampliación y reconocimiento de las necesidades individuales en torno a lo cual se genera un nicho de justicia, igualdad y universalidad, cada vez más interpelado por las personas migrantes, en tanto a su inclusión en las distintas sociedades destino.

\section{Referencias}

Acosta, D., \& Freier, L. (2015). Discursos y políticas de inmigración en Sudamérica. Revista Interdisciplinar da Mobilidade Humana, (44), 171-189.

Ballesteros, M. (2014). Un análisis sobre las desigualdades en el acceso a los servicios de salud en la población adulta urbana de la Argentina a partir de datos secundarios, Documentos de Jóvenes Investigadores, 4. Buenos Aires, Argentina: Instituto de Investigaciones Gino Germani, Facultad de Ciencias Sociales, UBA.

Basualdo, L. (2015). Inclusión diferencial de extranjeros/migrantes a trasplantes de órganos: dilemas éticos frente a "prácticas no éticas". Revista Interdisciplinar da Mobilidade Humana, 25(50), 65-80. https://doi.org/10.1590/198085852503880005005

Brumat, L. (2019). (Libre) circulación de personas y políticas migratorias regionales en el MERCOSUR. Estado del arte. Revista MERCOSUR de Politicas Sociales, 3, 10-24. https://doi.org/10.1590/198085852503880005005

Cabieses, B., \& Oyarte, M. (2017). Acceso a salud en inmigrantes: identificando brechas para la protección social en salud. Revista de Saúde Pública, (54), 1-13. https://doi.org/10.34052/rispch.vli1.17

Camarero Santamaría, Jesús (1998) El déficit social neoliberal. Del Estado de bienestar a la sociedad de la exclusión, España, Santander: Sal Térrea.

Canelo, B., Gavazzo, N., \& Nejamkis, L. (2018). Nuevas (viejas) políticas migratorias en la Argentina del cambio. Sí somos americanos. Revista de estudios transfronterizos, 18(1),150-182. https:// doi.org/10.4067/S0719-09482018000100150

Carrasco, I., \& Suárez, J. (2018). Migración internacional e inclusión en América Latina: Análisis en los paises de destino mediante encuestas de hogares. Santiago: Naciones Unidas. 
Castel, R. (2014). Los riesgos de exclusión social en un contexto de incertidumbre. Revista Internacional De Sociología, 72, 15-24. https://doi.org/10.3989/ ris.2013.03.18

Cecchini, S., Filgueira, F., Martínez, R. y Rossel, C. (2015) Instrumentos De Protección Social: Caminos Latinoamericanos hacia la universalización. Santiago de Chile: Naciones Unidas, Comisión Económica para América Latina y El Caribe (CEPAL)

Cerani, P. (2011). Luces y sombras en la legislación migratoria latinoamericana. Nueva Sociedad, (233), 68-86.

Courtis, C., Liguori, G., \& Cerruti, M. (2010). Migración y salud en zonas fronterizas: el Estado Plurinacional de Bolivia y la Argentina. Santiago: Naciones Unidas.

Domenech E., \& Pereira A. (2017). Estudios migratorios e investigación académica sobre las políticas de migraciones internacionales en Argentina. Íconos. Revista de Ciencias Sociales, (58), 83-108. https://doi.org/10.17141/iconos.58.2017.2487

Felder, F. (2018). The Value of Inclusion. Journal of Philosophy of Education, 52(1), 54-70. https://doi. org/10.1111/1467-9752.12280

Fraser, Nancy (2003) ¿Redistribution or Recognition? A POlitical-Philosophical Exchange. London: Verso Books.

García L. (2016) Migraciones, Estado y una política del derecho humano a migrar: ¿hacia una nueva era en América Latina? Colombia international. N88. pp. 107-133 http://dx.doi.org/10.7440/colombiaint88.2016.05

García L., \& Nejamkis, L. (2018). Regulación migratoria en la Argentina actual: del "modelo" regional al recorte de derechos. Autoctonía. Revista de Ciencias Sociales e Historia, 2(2), 219-241. https:// doi.org/10.23854/autoc.v2i2.55

Gíl Araujo y Santi, S. (2019). El gobierno de la migración en América del Sur: regímenes, contro-les y fronteras. Revista de Investigación sobre Migraciones, Periplos, 3(1), 2-22.

Goldberg A, Silveira C. (2013). Desigualdad social, condiciones de acceso a la salud pública y procesos de atención en inmigrantes bolivianos de Buenos Aires y São Paulo: una indagación comparativa. Saúde Soc, 22(2), 283-297. https://doi. org/10.1590/S0104-12902013000200003

Gutiérrez J., \& Hernández, M. (2013). Cobertura de protección en salud y perfil de la población sin protección en México, 2000 - 2012. Salud Publica Mex., 55(2), 83 - 90. https://doi.org/10.21149/ spm.v55s2.5102

Jaramillo, V. (2013) ¿Cuáles son los derechos humanos de las personas migrantes vigentes en Argenti- na? Universidad de Buenos Aires. Facultad de Ciencias Sociales. Instituto de Investigaciones Gino Germani; Argumentos; 15; 37-66.

Jelin, E. (2021). Género, etnicidad/raza y ciudadanía en las sociedades de clases. Realidades históricas, aproximaciones analíticas. Nueva Sociedad, (293), 39-62.

Piñones, Liberona, N., \& Mansilla, M. (2017). Perspectivas teóricas sobre salud y migración: determinantes sociales, transnacionalismo y vulnerabilidad estructural. Saude e Sociedade. São Paulo, v.30, n.1, p.19. https://doi.org/10.1590/ S0104-12902021200310

Maceira, D. (2020). Caracterización del Sistema de Salud Argentino:Debate latinoamericano. Revista Estado y Políticas Públicas, (14), 155-179.

Manchola, C., Garrafa V., Cunha T., \& Hellmann F. (2017). $\mathrm{El}$ acceso a la salud como derecho humano en políticas internacionales: reflexiones críticas y desafíos contemporáneos. Ciênc. saúde colet, 22(7), 2151-2160. https://doi.org/10.1590/1413$\underline{81232017227.04472017}$

Mannoukian, D., \& Venturini, N. (2021). Sistema de salud. El modelo argentino y el caso neuquino. Buenos Aires: CICCUS.

Mario, S. (2018). Servicios de salud: cobertura, acceso y utilización. En J. Piovani, y A. Salvia, (coord.), La Argentina del siglo XXI. Cómo somos, vivimos y convivimos en una sociedad desigual (pp.265-290). Buenos Aires: Siglo XXI editores.

Martínez, R y Carpinetti, E (2021) Caracterización sociodemográfica y ocupacional de la migración reciente a la Ciudad de Buenos Aires. Población \& Sociedad, Vol. 28 (1), pp. 53-85. http://dx.doi. org/10.19137/pys-2021-280104

Molina, C. (2019). La salud de los migrantes como desafío para la salud pública. Revista de Trabajo Social y Ciencias Sociales, 29, 1-8.

Muñoz, B., \& Barrantes, A. (2016). Equidad e Inclusión Social: Superando desigualdades hacia sociedades más inclusivas. OEA.

Nicolao, J. (2010). Los migrantes regionales en Bahía Blanca, Argentina: Desafíos en el acceso a derechos sociales. Revista de Estudios Fronterizos, 20, 1-25. https://doi.org/10.21670/ref.1903024

Novick, S. (2010). Migraciones y Mercosur: una relación inconclusa. En: Migración y políticas públicas. Nuevos escenarios y desafios (p.165). Buenos Aires: Catálogos-CLACSO.

Oszlak, Oscar y O’Donnell, Guillermo 1976 Estado y politicas estatales en América Latina: hacia una estrategia de investigación. Buenos Aires: Documento CEDES / G. E. CLACSO. $\mathrm{N}^{\circ} 4$.

Padrón, M \& Román, P. Exclusión social y exclusión en salud: apuntes teórico-conceptuales y metodológicos para su estudio social. Boletín Mex- 
icano de Derecho Comparado, nueva serie, año XLIII, núm. 128, mayo-agosto de 2010, pp. 815-843.

Penchaszadeh, A. (2012). Migraciones y derechos políticos: ¿democratización y extensión de la ciudadanía o nuevas formas de la extranjerización en democracia? En: Migración y políticas públicas. Nuevos escenarios y desafíos. (39-62). Buenos Aires: Catálogos-CLACSO.

Piñones, C., Liberona, N., \& Leiva, S. (2021). Perspectivas teóricas sobre salud y migración: determinantes sociales, transnacionalismo y vulnerabilidad estructural. Saude e Sociedade, 30(1), 3-18. https://doi.org/10.1590/s0104-12902021200310

Rodríguez, S., Schiavone, M., Seoane, H., \& Ríos, J. (2015). Salud percibida, cobertura, utilización y acceso al sistema de atención de la salud en el área metropolitana de Buenos Aires, Fundación Universidad Católica Argentina.

Thayer, L., \& Durán, C. (2015). Gobierno local y migrantes frente a frente: Nudos críticos y políticas para el reconocimiento. Revista del CLAD Reforma y Democracia, (63), 127-162.

Thayer, L., \& Stang, F. (2017). Estatus legal precario y condicionalidad en el acceso a derechos. Una aproximación a la regulación migratoria de Argentina y Canadá. Revista de Estudios Transfronterizos. Si Somos Americanos. (12), 11-43. http://dx.doi.org/10.4067/S0719-09482016000200001 